\title{
Magnifying i-scan Imaging for Endoscopic Diagnosis of Helicobacter pylori Infection: A Prospective Study
}

\author{
Avinash Bhat Balekuduru ${ }^{1}$ Aarathi Rau² \\ Satyaprakash Bonthala Subbaraj' \\ ${ }^{1}$ Department of Gastroenterology, M.S. Ramaiah Medical College, \\ Bengaluru, Karnataka, India \\ 2Department of Pathology, M.S. Ramaiah Medical College, \\ Bengaluru, Karnataka, India
}

\begin{abstract}
Address for correspondence Avinash Bhat Balekuduru, MD, DNB, DM, Department of Gastroenterology, M.S. Ramaiah Medical College, Bengaluru, Karnataka 560054, India (e-mail: avinashbalekuduru@gmail.com).
\end{abstract}

J Digest Endosc 2020;11:112-117

\begin{abstract}
Keywords

- i-scan

- Helicobacter pylori gastritis

- magnifying white light endoscopy

Background DEFINA (EPK 3000) plus (with i-scan) is a digital chromoendoscopy. It uses surface/tone/contrast enhancement for detection, demarcation, and characterization of lesions. The aim of this study was to compare the usefulness of i-scan and conventional magnification white light endoscopy (M-WLE) for diagnosing Helicobacter pylori ( $\mathrm{Hp})$ infection in stomach.

Patients and Methods Subjects undergoing evaluation for functional dyspepsia were prospectively enrolled at Ramaiah Medical College and Hospitals, Bangalore from November 2018 to February 2019. In total, 68 participants underwent gastroscopy with standard M-WLE followed by i-scan. Two biopsies from greater curve at $3 \mathrm{~cm}$ from the angulus were collected for histology. Successful diagnosis of Hp using imaging modality with M-WLE and i-scan were compared with histology.

Results A total of 68 ( 36 men and 32 women) patients with a mean age of $47 \pm 13$ years (range 18-75 years) were enrolled in our study. The prevalence of $\mathrm{Hp}$ on the biopsies was $41 \% ; 64 \%$ of the patients used proton pump inhibitors, $20 \%$ were current smokers; $25 \%$ of the patients were consuming alcohol. The sensitivity: $96.4 \%$; specificity: $95 \%$; accuracy: $95.5 \%$ of $\mathrm{i}$-scan in diagnosis of $\mathrm{Hp}$ gastritis is better than the sensitivity: $50 \%$; specificity: $50 \%$; accuracy: $50 \%$ with M-WLE.

Conclusion In conclusion, the diagnostic ability of $\mathrm{i}-\mathrm{scan}$ (95\%) for predicting $\mathrm{Hp}$ status is acceptable as compared with M-WLE (50\%) for accurate diagnosis. The results suggest that i-scan improves endoscopic diagnostic accuracy of Hp infection compared with M-WLE.
\end{abstract}

\section{Introduction}

The mortality of gastric cancer (GC) has been decreasing worldwide, but there are still more than 800,000 deaths due to this disease. GC is the third leading cause of cancer death worldwide. In most cases, it is detected at an advanced clinical stage and has a poor overall 5-year survival ${ }^{1,2}$

Helicobacter pylori (Hp) is the most prevalent chronic bacterial infection. Hp is associated with peptic ulcer disease, chronic gastritis, gastric adenocarcinoma, and gastric mucosa-associated lymphoid tissue lymphoma. ${ }^{3-6}$
Noninvasive tests (e.g., serology, urea breath test, or stool test) are convenient and accurate. However, these tests do not provide real-time information on the gastric mucosa and time consuming. ${ }^{7}$

The multistep model of gastric carcinogenesis of $\mathrm{Hp}$ is by chronic inflammation of the gastric mucosa, progressing through the chronic atrophic gastritis (CAG), intestinal metaplasia (IM)/dysplasia, intramucosal carcinoma, and invasive neoplasia. ${ }^{8}$ Conventional magnification white light endoscopy (M-WLE) frequently correlates poorly with histopathological findings of Hp gastritis. ${ }^{9}$ Using M-WLE, features of diffuse redness in the corpus and mucosal swelling in
License terms

(우 (1) $\ominus \circledast$ 
the antrum were shown to be hallmarks of Hp infection. ${ }^{9}$ Diffuse redness of the fundic mucosa was found on significant tissue area in Hp-positive patients (failed eradication) than in Hp-negative (successful eradication) patients in a multicenter prospective trial. ${ }^{10}$ The diagnostic performance of image-enhanced endoscopy (IEE) techniques such as narrow-band imaging (NBI), i-scan for diagnosing Hp on endoscopy have been reported. ${ }^{11,12}$ CAG and IM are the earliest phenotypic markers in the gastric carcinogenic sequence, and surveillance will depend on histological confirmation of those lesions.

$\mathrm{i}$-scan is a dynamic, software-based, image enhancement technology that provides an enhanced view of the texture of the mucosal surface and blood vessels by performing per pixel modifications of the white light image.

Random biopsies are not always sufficiently accurate for the detection of Hp due to its focal distribution. Esophagogastroduodenoscopy (EGD) examinations and a biopsy from the gastric mucosa are required for these patients, which have risks such as missing pathology and sampling errors and is expensive. Therefore, the development of real-time endoscopic assessments for the diagnosis of $\mathrm{Hp}$ during endoscopy has emerged. The ultimate goal of endoscopy will be "optical biopsy," that is, forgoing the need to take histological samples to make a diagnosis to reduce complication rates, cost, and time. This study aims to compare the diagnostic value of M-WLE, i-scan with histology to diagnose Hp infection in subjects with functional dyspepsia (FD).

\section{Methods}

\section{Sample Size Calculation}

Sample size was estimated by considering the sensitivity and specificity of high definition M-WLE to diagnose Hp infection, which was 95 and $90 \%$, respectively ${ }^{13}$ with an $\alpha$ error considered as $5 \%$ and relative precision as $8 \%$ (by using $n$ master software). The minimum number of patients to be included was 54 .

\section{Subjects}

Study participants with the diagnosis of FD (Rome IV) ${ }^{14}$ were prospectively enrolled at Ramaiah Medical College and Hospitals, Bangalore from November 2018 to February 2019. In total, 68 participants who underwent upper gastroscopy for FD had standard M-WLE followed by i-scan and histology.

Two biopsies from greater curve/corpus at $3 \mathrm{~cm}$ from the angulus were collected from all patients using single-use radial jaw forceps (Boston Scientific, Natick, Massachusetts, United States) as per hospital standard of practice.

The biopsies were placed together in a vial pod in 10\% formaldehyde bottles.

Medical history including proton pump inhibitors (PPI) intake, tobacco, and alcohol, a careful collection of images of M-WLE, i-scan on presence or absence of $\mathrm{Hp}$ and histological findings were recorded on a uniform structured proforma.

\section{Endoscopic Procedure}

The video endoscope used in the present study was a forward-viewing single-channel high definition gastroscope (PENTAX/EG 2990K) (Pentax Medical Singapore) with a $9.8-\mathrm{mm}$ outer diameter insertion tube with up to 2.8-mm channel diameter; using a light source video processor PENTAX/EPK-3000 (DEFINA plus) (Pentax Medical Singapore), the magnification up to $2 \times$ (200 times) is achieved. Magnification is achieved easily through a manual adjustment switch on the endoscopic handle by the endoscopist. The enhancement function can be changed by touch screen operation. A transparent cap is attached to the distal tip of the endoscope to maintain focal distance during the magnifying observation.

A conscious sedation was performed at the request of the patient. Four hours before the procedure, all patients ingested $140 \mathrm{mg}$ of simethicone diluted in $40 \mathrm{~mL}$ of water (A. Menarini India Pvt. Limited, Elphinstone road [W], Mumbai). After the endoscope was inserted, the entire stomach was initially observed with conventional M-WLE to exclude obvious lesions. Later i-scan 3 was performed for more detailed topography of the mucosal surface and vascularity to detect $\mathrm{Hp}$.

After a routine examination of the entire stomach by high definition endoscopy, the greater curvature of the gastric corpus was carefully observed under complete magnification ( $\times 200$ ), using WLE (without TE [tone enhancement], SE [surface enhancement], and CE [contrast enhancement]) and i-scan 3 (with gastric TE mode [g TE], $+2 \mathrm{SE}$ and $+4 \mathrm{CE}$ [representing the proper level of magnification for good visualization among different enhancements of $\mathrm{SE}$ and $\mathrm{CE}$ modes]), respectively.

i-scan 3 images were categorized into three types based on subepithelial capillary network (SECN), collecting venules (CV), and pits. Type 1 ( - Fig. 1), honeycomb-type SECN with regular arrangement of $\mathrm{CV}$ (spider like pattern) and regular, round pits, Type 2 (-Fig. 2) honeycomb-type SECN with slightly enlarged pits, but loss of CV and Type 3 (-Fig. $\mathbf{3}$ ). Coiled or wavy SECN and loss of CV, with oval or prolonged pits surrounded by increased density of irregular vessels. Type 1 was noted as Hp negative and types $2 / 3$ were noted at Hp positive. ${ }^{15,16}$

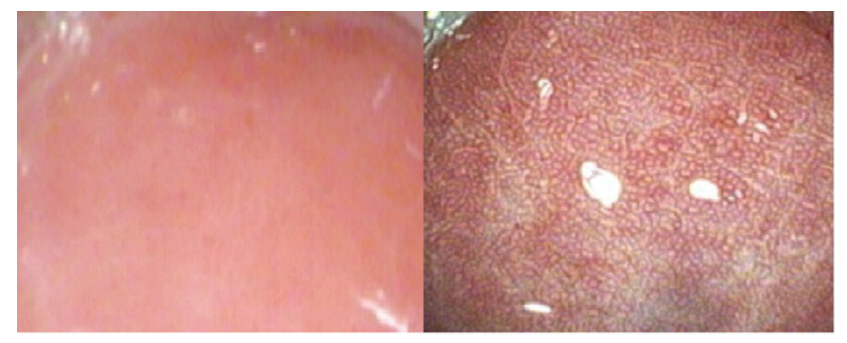

Fig. 1 Picture depicting Type 1: Honeycomb-type SECN with regular arrangement of CV (spider like pattern) and regular, round pits-on M-WLE and i-scan. CV, collecting venules; M-WLE, magnifying white light endoscopy; SECN, subepithelial capillary network. 


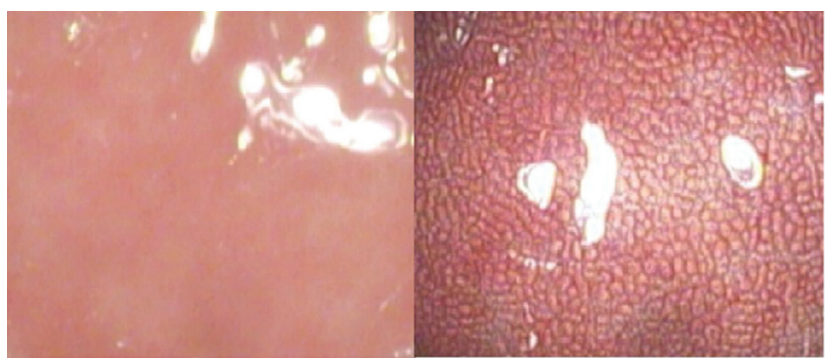

Fig. 2 Picture depicting Type 2: Honeycomb-type SECN with slightly enlarged pits, but loss of CV on M-WLE and i-scan. CV, collecting venules; M-WLE, magnifying white light endoscopy; SECN, subepithelial capillary network.

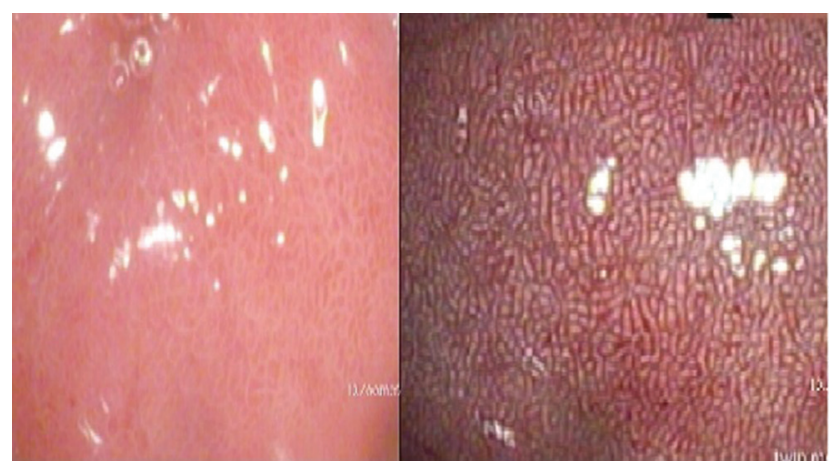

Fig. 3 Picture depicting Type 3: coiled or wavy SECN and loss of $\mathrm{CV}$, with oval or prolonged pits surrounded by increased density of irregular vessels-on M-WLE and i-scan. CV, collecting venules; M-WLE, magnifying white light endoscopy; SECN, subepithelial capillary network..

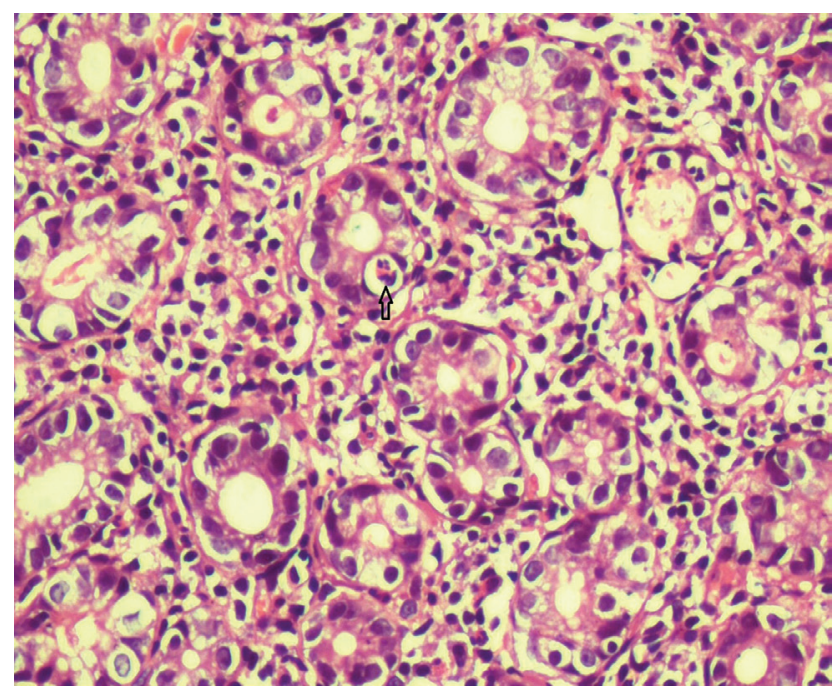

Fig. 4 Picture depicting HPE of $\mathrm{H}$ and $\mathrm{E}$ staining-400X-showing active $\mathrm{Hp}$ gastritis. Hp, Helicobacter pylori.

\section{Histological Examination}

Hematoxylin and eosin staining was performed. Specimens were also screened for $\mathrm{Hp}$ infection using a modified $2 \%$ Giemsa stain (-Figs. 4-6). Neither clinical information nor endoscopic findings were blinded to the pathologist.

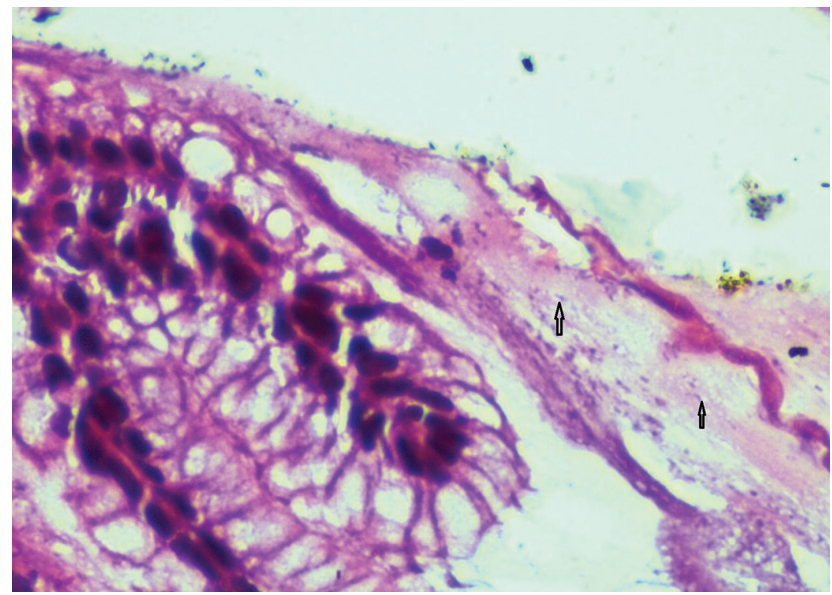

Fig. 5 Picture depicting HPE of $\mathrm{H}$ and E staining-1000X showing $\mathrm{Hp}$. Hp, Helicobacter pylori.

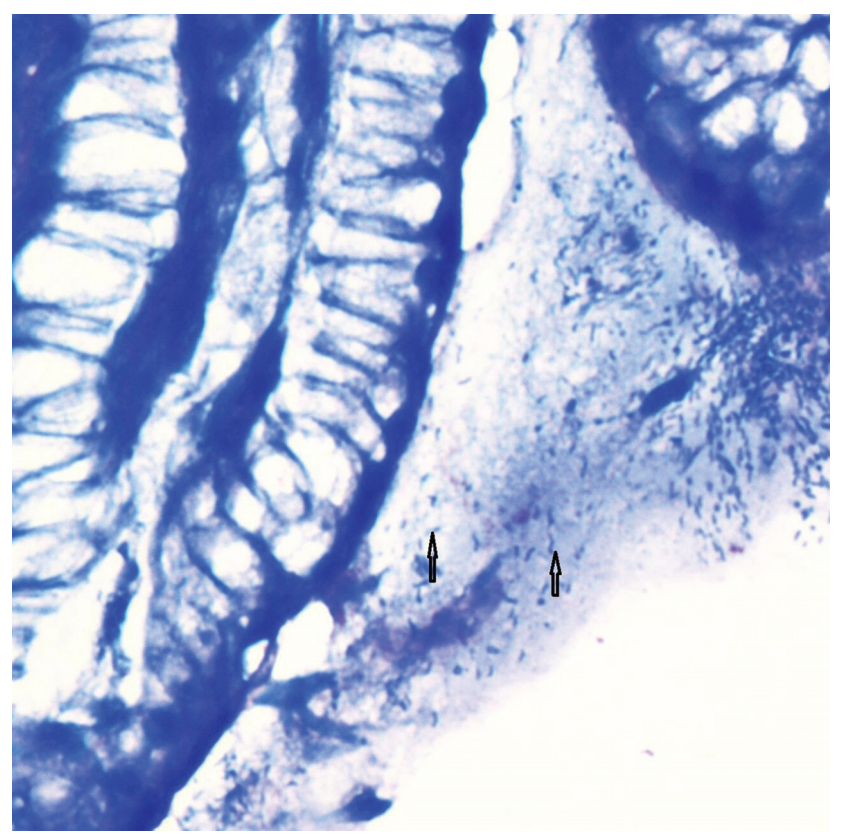

Fig. 6 Picture depicting HPE of modified 2\% Giemsa staining-1000X showing Hp. Hp, Helicobacter pylori.

\section{Primary Outcome Measures}

Successful diagnosis of Hp using imaging modality with M-WLE and i-scan was compared with histology.

\section{Inclusion Criteria}

All FD patients above the age of 18 years attending for a routine diagnostic endoscopic procedure at Ramaiah Medical College and Hospitals, Bangalore were included in the study.

\section{Exclusion Criteria}

Patients attending for a (1) therapeutic endoscopic procedure (2) age $<18$ years or $>80$ years; (3) with severe systemic diseases; (4) the use of antibiotics bismuth compounds, PPIs, or $\mathrm{H} 2$ receptor antagonist within 6 weeks before the endoscopic procedure; (5) previous Hp eradication therapy; (6) the use of 
nonsteroidal anti-inflammatory drugs; (7) a history of gastric surgery; (8) severe coagulopathy; (9) pregnancy or lactation, and (10) unwilling to give informed consent.

The Institute ethical committee approved the protocol and written informed consent was obtained from all participants to undergo EGD using both M-WLE and i-scan.

\section{Statistical Analysis}

The baseline characteristics were expressed as mean with standard deviation for continuous data and as percentage for categorical data. Diagnostic performance of i-scan for diagnosing Hp was estimated. The sensitivity, specificity, diagnostic accuracy, disease prevalence were calculated. The SPSS 19.0 statistics program was used to calculate the values of the variables.

\section{Results}

A total of 68 patients ( 36 men and 32 women) with a mean age of $47 \pm 13$ years (range 18-75 years) were enrolled in our study. All the patients complained of dyspeptic symptoms such as abdominal pain, nausea, and poor appetite, received both M-WLE and i-scan examinations of the greater curvature of the stomach by complete magnification (2×). No complications like hemorrhage or perforation were observed during the endoscopic procedure

The demographic and clinical characteristics of the patients are shown in -Table $\mathbf{1}$.

Table 1 Showing the demographic and clinical characteristics of the patients

\begin{tabular}{|l|l|}
\hline Demography & Value \\
\hline Age (mean \pm SD) & $47 \pm 13 \mathrm{y}$ \\
\hline Sex (male:female) & $36(53 \%): 32(47 \%)$ \\
\hline $\begin{array}{l}\text { Hp on histology } \\
\text { Positive: Negative }\end{array}$ & $28(41.1 \%): 40(58.9 \%)$ \\
\hline Smoking & \\
\hline $\begin{array}{l}\text { Nonsmoker; Current: former } \\
\text { smoker }\end{array}$ & $47(70 \%): 13(20 \%): 8(10 \%)$ \\
\hline Alcohol & 51(75\%):17(25\%) \\
\hline Nondrinker: current drinker & $44(64.7 \%): 24(35.3 \%)$ \\
\hline Medication & \\
\hline PPI use: no use of PPI &
\end{tabular}

Abbreviations: PPI, proton pump inhibitors; SD, standard deviation.
The prevalence of Hp on the biopsies was 41\%; in addition, $64 \%$ of the patients used PPIs, $20 \%$ were current smokers, and $25 \%$ of the patients were consuming alcohol.

\section{Relationship between Magnifying i-scan and Hp Infection}

Hp infection was detected in $28(41.1 \%)$ participants. The relationship between the magnifying $\mathrm{i}$-scan gastric mucosal patterns and Hp infection is shown in - Tables 2 and 3. The sensitivity, specificity, and accuracy of magnifying i-scan and Hp infection were noted to be $96.4,95,95.5 \%$, respectively.

\section{Relationship between M-WLE and Hp Infection}

On M-WLE, endoscopic findings were noted as normal in 34 (50\%), gastric erosions in 21 (31\%), gastroduodenal erosions in 9 (13\%), and ulcer in $4(6 \%)$. The relationship between the WLE gastric mucosal patterns and Hp infection is shown in - Table 4. The sensitivity, specificity, and accuracy were noted to be 50,50 , and $50 \%$, respectively.

\section{Discussion}

Conventional EGD is generally used for Hp detection based on gross appearance of the gastric mucosa such as redness, mucosal swelling, and nodular changes, but these features are not specific enough for diagnosis. ${ }^{17}$

Histopathology is considered as gold standard for detecting Hp. ${ }^{18}$ Indeed, it has been reported that M-WLE itself has limited ability to distinguish $\mathrm{Hp}$-infected subjects. ${ }^{19}$ It is essential to use IEE such as NBI and i-scan for the endoscopic diagnosis of Hp infection.

Several endoscopic features are suggestive of chronic Hp gastritis, including the absence of CVs, antral nodularity, enlarged gastric folds, enlargement and destruction of the gastric glands, sticky tenacious adherent mucous, turbid gastric juice, and xanthomas ${ }^{20-23}$ Loss of CVs and capillary vascular structure was correlated with chronic inflammation and activity. With progression of mucosal atrophy, irregular CVs become visible. ${ }^{24}$

The field of IEE continues to progress rapidly. i-scan with optical enhancement applies digital image processing algorithms, by an optical filter which provides peak luminescence at the peaks of the hemoglobin absorption spectrum. i-scan is a digital chromoendoscopy technology with usage of SE, TE, and CE for detection, characterization, and demarcation of abnormalities in gastrointestinal tract. ${ }^{25,26}$ But, in practice, more training/time and experience of the endoscopist is required which limits the use of IEE.

Table 2 Showing the i-scan gastric mucosal patterns and Hp infection

\begin{tabular}{|l|l|l|l|}
\hline i-scan- $\boldsymbol{n}(\%)$ & Positive-28 (41.1\%) & Negative-40 (58.9\%) & Total-68 (100\%) \\
\hline Type 1 & $1(3 \%)$ & $38(95 \%)$ & $39(57.3 \%)$ \\
\hline Type 2 & $17(61 \%)$ & $1(2.5 \%)$ & $18(26.4 \%)$ \\
\hline Type 3 & $10(36 \%)$ & $1(2.5 \%)$ & $11(16.2 \%)$ \\
\hline Types 2 and 3 & $27(97 \%)$ & $2(5 \%)$ & $29(42.6 \%)$ \\
\hline
\end{tabular}

Abbreviation: Hp, Helicobacter pylori. 
Table 3 Showing combined i-scan 2 and 3 comparison to type 1 and Hp infection

\begin{tabular}{|l|l|l|l|}
\hline $\mathrm{Hp}$ & Histology positive & Histology negative & Total \\
\hline i-scan 2/3 positive & 27 & 2 & 29 \\
\hline i-scan 1 negative & 1 & 38 & 39 \\
\hline Total & 28 & 40 & 68 \\
\hline
\end{tabular}

Abbreviation: Hp, Helicobacter pylori.

Table 4 Showing the M-WLE gastric mucosal patterns and Hp infection

\begin{tabular}{|l|l|l|l|}
\hline Hp M-WLE & Histology positive & Histology negative & Total \\
\hline Positive (Types 2 and 3) & 14 & 20 & 34 \\
\hline Negative (Type 1) & 14 & 20 & 34 \\
\hline Total & 28 & 40 & 68 \\
\hline
\end{tabular}

Abbreviations: Hp, Helicobacter pylori; M-WLE, magnifying white light endoscopy

The use of PPIs may decrease the sensitivity of detection of $\mathrm{Hp}$ by urea breath test. ${ }^{27}$ There has been an increase in the use of PPIs, antiplatelet, and anticoagulants in clinical practice. Biopsy for evaluation of Hp can carry a risk of bleeding. Therefore, the magnifying i-scan system would play an important role for patients undergoing such medications. We assessed i-scan mucosal patterns in the greater curvature of the gastric body as gastric atrophy would be mild in the stomach. ${ }^{26}$ We did not choose the antrum as targeted part because the $\mathrm{CV}$ is difficult to be observed due to its location.

Hp-negative gastric mucosa was characterized as small, round pits, accompanied with regular honeycomb-like SECNs, being regularly interspersed with CV (type 1). When the stomach is infected with Hp edema, destruction and neovascularization caused by inflammation enlarge the pits and make the capillaries and venules invisible. Hp-positive gastric mucosa was characterized as various sizes of enlarged or elongated pits with unclear SECNs or dense fine irregular vessels (types $2 / 3$ ). ${ }^{27,28}$

Yagi et al reported a sensitivity of $93.8 \%$ and a specificity of $96.2 \%$ of the regular arrangement of CV for Hp-negative normal gastric mucosa. ${ }^{26}$ In our study, sensitivity of $96.4 \%$ and specificity of $95 \%$ were noted for i-scan types $2 / 3$ in diagnosing $\mathrm{Hp}$ positive gastritis in gastric body. The pooled sensitivity and specificity using pit and vascular pattern in gastric corpus was 96 and 91\%, respectively in a meta-analysis. ${ }^{29}$ Magnification endoscopy was able to accurately indicate the status of Hp infection, in both magnifying WLE and chromoendoscopy mode. Furthermore, the diagnostic efficiency was better when targeting the gastric corpus than the antrum. Pit plus vascular pattern classification in the corpus was the optimum diagnostic criteria. ${ }^{29}$ The current study had taken gastric corpus for detecting $\mathrm{Hp}$.

In our study there were two patients whose mucosal patterns were types 2-3 (the indicator of $\mathrm{Hp}$ ) under i-scan; they were all Hp negative. These two patients might have had alcohol/smoking-induced gastritis. There were two cases of gastric atrophy noted on histology but missed on i-scan-there might have been focal atrophy. As the major aim of our study was to determine the relationship between magnifying endoscopic patterns and $\mathrm{Hp}$ infection we did not analyze this kind of endoscopic pattern. There were no dysplasia/cancer in the study. In our study, there were five cases of mild IM on histology which could not be noted on i-scan. Furthermore, Hp infection was determined on the basis of mucosal changes caused by inflammation. For patients without inflammation after $\mathrm{Hp}$ infection or with inflammation that is not related to $\mathrm{Hp}$ infection, our results might not be applicable

Our study has limitations. First, the number of patients was small and the data are from a single center. Second, gastric body was examined in our study. Hp inflammation can be patchy and sample bias is a possibility. Multiple diagnostic tests might have been used for the diagnosis. Third, the relationship between i-scan and the updated Sydney system was not assessed in this study. Fourth we did not assess the interobserver and intraobserver agreement, which restricted the reproducibility of this study. Fifth, therefore, M-WLE and i-scan examination combined with the updated Sydney system will be needed for further multi-institutional studies with a large number of cases.

In summary, the typical pattern used to predict an $H$. pylori negative gastric mucosa is featured as honeycomb-type SECN with regular arrangement of CVs and regular, round pits. In the $H$. pylori positive gastric mucosa, the CVs become invisible and gastric pits are enlarged, with SECN irregular or disappeared. i scan of gastric corpus is an optimum endoscopic criterion for $H$. pylori infection in clinical practice. The diagnostic ability of i-scan (95\%) for predicting Hp status significantly improved the endoscopic diagnostic accuracy as compared with M-WLE (50\%) in our study.

\section{Conflict of Interest}

None declared. 


\section{References}

1 Fujiyoshi T, Miyahara R, Funasaka K, et al. Utility of linked color imaging for endoscopic diagnosis of early gastric cancer. World J Gastroenterol 2019;25(10):1248-1258

2 Ferlay J, Soerjomataram I, Dikshit R, et al. Cancer incidence and mortality worldwide: sources, methods and major patterns in GLOBOCAN 2012. Int J Cancer 2015;136(5):E359-E386

3 NIH Consensus Conference. Helicobacter pylori in peptic ulcer disease. NIH consensus development panel on Helicobacter pylori in peptic ulcer disease. JAMA 1994;272(1):65-69

4 Malfertheiner P, Megraud F, O'Morain CA, et al. European Helicobacter Study Group. Management of Helicobacter pylori infection-the Maastricht IV/Florence consensus report. Gut 2012;61(5):646-664

5 Chey WD, Wong BC; Practice Parameters Committee of the American College of Gastroenterology. American College of Gastroenterology guideline on the management of Helicobacter pylori infection. Am J Gastroenterol 2007;102(8):1808-1825

6 Laine L. Helicobacter pylori, gastric ulcer, and agents noxious to the gastric mucosa. Gastroenterol Clin North Am 1993;22(1):117-125

7 Chen TH, Hsu CM, Cheng HT, et al. Linked color imaging can help gastric Helicobacter pylori infection diagnosis during endoscopy. J Chin Med Assoc 2018;81(12):1033-1037

8 Busuttil RA, Boussioutas A. Intestinal metaplasia: a premalignant lesion involved in gastric carcinogenesis. J Gastroenterol Hepatol 2009;24(2):193-201

9 Dohi O, Yagi N, Onozawa Y, et al. Linked color imaging improves endoscopic diagnosis of active Helicobacter pylori infection. Endosc Int Open 2016;4(7):E800-E805

10 Kato M, Terao S, Adachi K, et al; Study Group for Establishing Endoscopic Diagnosis of Chronic Gastritis. Changes in endoscopic findings of gastritis after cure of H.pylori infection: multicenter prospective trial. Dig Endosc 2013;25(3):264-273

11 Nakayoshi T, Tajiri H, Matsuda K, Kaise M, Ikegami M, Sasaki H. Magnifying endoscopy combined with narrow band imaging system for early gastric cancer: correlation of vascular pattern with histopathology (including video). Endoscopy 2004;36(12):1080-1084

12 Ezoe Y, Muto M, Uedo N, et al. Magnifying narrowband imaging is more accurate than conventional white-light imaging in diagnosis of gastric mucosal cancer. Gastroenterology 2011;141(6):2017-2025.e3

13 Anagnostopoulos GK, Yao K, Kaye P, et al. High-resolution magnification endoscopy can reliably identify normal gastric mucosa, Helicobacter pylori-associated gastritis, and gastric atrophy. Endoscopy 2007;39(3):202-207

14 Stanghellini V, Chan FK, Hasler WL, et al. Gastroduodenal disorders. Gastroenterology 2016;150(6):1380-1392

15 Tahara T, Shibata T, Nakamura M, et al. Gastric mucosal pattern by using magnifying narrow-band imaging endoscopy clearly distinguishes histological and serological severity of chronic gastritis. Gastrointest Endosc 2009;70(2):246-253
16 Nakagawa S, Kato M, Shimizu Y, et al. Relationship between histopathologic gastritis and mucosal microvascularity: observations with magnifying endoscopy. Gastrointest Endosc 2003;58(1):71-75

17 Kato T, Yagi N, Kamada T, Shimbo T, Watanabe H, Ida K; Study Group for Establishing Endoscopic Diagnosis of Chronic Gastritis. Diagnosis of Helicobacter pylori infection in gastric mucosa by endoscopic features: a multicenter prospective study. Dig Endosc 2013;25(5):508-518

18 Correa P, Piazuelo MB, Wilson KT. Pathology of gastric intestinal metaplasia: clinical implications. Am J Gastroenterol 2010;105(3):493-498

19 Yagi K, Saka A, Nozawa Y, Nakamura A. Prediction of Helicobacterpyloristatus by conventional endoscopy, narrow-band imaging magnifying endoscopy in stomach after endoscopic resection of gastric cancer. Helicobacter 2014;19(2):111-115

20 Yagi K, Aruga Y, Nakamura A, Sekine A. Regular arrangement of collecting venules (RAC): a characteristic endoscopic feature of Helicobacter pylori-negative normal stomach and its relationship with esophago-gastric adenocarcinoma. J Gastroenterol 2005;40(5):443-452

21 Yagi K, Honda H, Yang JM, Nakagawa S. Magnifying endoscopy in gastritis of the corpus. Endoscopy 2005;37(7):660-666

22 Yagi K, Nakamura A, Sekine A. Comparison between magnifying endoscopy and histological, culture and urease test findings from the gastric mucosa of the corpus. Endoscopy 2002;34(5):376-381

23 Gonen C, Simsek I, Sarioglu S, Akpinar H. Comparison of high resolution magnifying endoscopy and standard videoendoscopy for the diagnosis of Helicobacter pylori gastritis in routine clinical practice: a prospective study. Helicobacter 2009; 14(1):12-21

24 Banks M, Graham D, Jansen M, et al. British Society of Gastroenterology guidelines on the diagnosis and management of patients at risk of gastric adenocarcinoma. Gut 2019;68(9):1545-1575

25 Tongtawee T, Dechsukhum C, Leeanansaksiri W, et al. Improved detection of Helicobacter pylori infection and premalignant gastric mucosa using "site specific biopsy": a randomized control clinical trial. Asian Pac J Cancer Prev 2015;16(18): $8487-8490$

26 Yagi K, Nakamura A, Sekine A. Magnifying endoscopy of the gastric body: a comparison of the findings before and after eradication of Helicobacter pylori. Dig Endosc 2002;14:S76-S82

27 Shirin H, Frenkel D, Shevah O, et al. Effect of proton pump inhibitors on the continuous real time (13)C-urea breath test. Am J Gastroenterol 2003;98(1):46-50

28 Qi QQ, Zuo XL, Li CQ, et al. High-definition magnifying endoscopy with i-scan in the diagnosis of Helicobacter pylori infection: a pilot study. J Dig Dis 2013;14(11):579-586

29 Qi Q, Guo C, Ji R, Li Z, Zuo X, Li Y. Diagnostic performance of magnifying endoscopy for Helicobacter pylori infection: a meta-analysis. PLoS One 2016;11(12):e0168201 